\section{QUERY: An interactive computer program for the collection of checklist data}

JAMES W. LIVINGSTON, JR.

and

\author{
SAMUEL J. KINGSLEY \\ Rutgers Cniversit. New Brunswick, New Jerse, 08903
}

QUERY is an interactive computer program that can be used to present an automated checklist or to automatically record data. The program operates on rectangular data matrices. where the rows may represent any objects at all and the columns represent characteristics of rows. For example, the matrix may represent people and traits, taxonomic units and taxonomic characteristics (Sneath \& Sokal. 1973), or cases and psychiatric sy'mptoms.

QUERY presents all the characteristics in sequence for the first row, then repeats this procedure for the second row. and so on, until all the rows are exhausted. The characteristics are presented one at a time so that the user may rate to what degree each applies to the row. QUERY presents the next characteristic as soon as the user enters a rating. The ratings presently recognized by QUERY are " 0 ," " 1 ," and " 2 ."

The ADD command makes it possible to add new characterstics to the list. If a new characteristic is added, the rows already presented will be re-presented for judgment on the new characteristic. The re-presentation occurs either (a) the next time that QUERY is run or (b) when all of the rows have been exhausted.

After a response is made to a characteristic, QUERY reviews that characteristic to determine the frequency of " 0 ," " 1 ," and " 2 " responses. Those characteristics which have a high proprotion of $0 \mathrm{~s}$ are deleted. The proportion currently used is $95 \%$.

When the data matrix is of moderate size, the user may not wish to complete the checklist in one session. To permit the user to save whatever has been completed, QUERY writes the data matrix on a disk file when the user requests. At each session, QUERY begins by reading the disk file. The auxiliary programs in the package are for the creation and maintenance of the disk files used by QUERY.

The use of QUERY in collecting person-perception data has been reported by Rosenberg (1976). In this application. people are regarded as rows, and traits are regarded as columns. Below is an abbreviated session of person description.

Iun utility

UTILITY $\quad 11: 00 \quad 11 / 12 / 75$

FILE

?tsk

TYPE OF FILE-TRAIT OR FEELING

?trait

YOUR INITIALS

?sk

TRAITS TO BE ADDED

?politician

?violent

?liberal

?attractivic

?done
YOUR FILE HAS BEEN UPDATED.

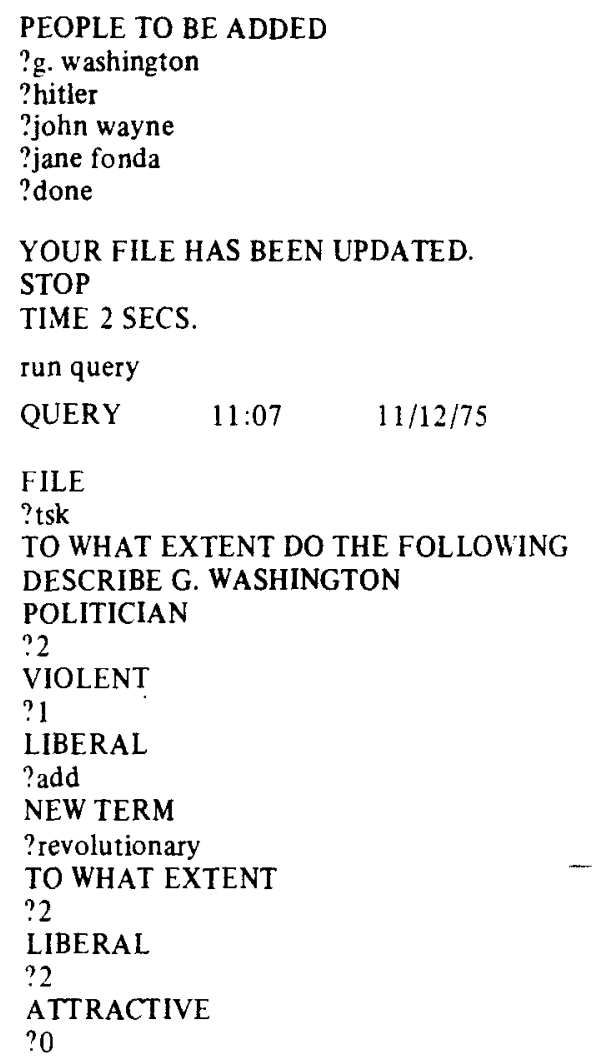

TO WHAT EXTENT DO THE FOLLOWING DESCRIBE HITLER POLITICIAN ?2 VIOLENT ?2 LIBERAL

?done YOUR FILE HAS BEEN UPDATED. STOP TIME 2 SECS.

The checklist construction program, UTILITY, performs the operations of entering a list of row labels (names of people) and/or a list of column labels (traits). Traits may also be added as the subject progresses through the checklist. After people names and trait terms are entered, UTILITY constructs a matrix with a row for each person and a column for each trait. At the outset, the matrix is filled with $9 \mathrm{~s}$, indicating that the cell has not yet been rated. The matrix is stored in a file, followed by the list of people and the list of traits.

In some circumstances, it is desirable to create separate groups of characteristics. For example, categorical variables must sometimes be distinguished from quantitative variables; test scores from time ${ }_{1}$ must be distinguished from the same tests administered at time ${ }_{2}$, time ${ }_{3}$, or time $\mathbf{k}_{\mathbf{k}}$, and demographic characteristics must be separated from test scores. Besides traits of people, the subjects in the person-perception experiment described the feelings elicited by the people. Two files were used for each subject: one for traits and one for feelings. In general, the programs can create any number of files with the same rows and different columns.

The auxiliary programs in the package are mainly for file manipulation. These are the "core" programs used in our 
research, although we have numerous variations on these that serve particular purposes.

REPLACE is the general-purpose error-correction program. It can: (a) change the type designation of a file from trait to feeling, or vice versa, (b) change the name of a descriptor (trait or feeling) or person, or (c) delete a descriptor (trait or feeling) or person.

LIST is a program to list a file at the terminal.

DUPE makes a duplicate of a file (particularly necessary in an unreliable system).

PCOPY permits the person list to be copied into an empty file and the type of descriptors (traits or feelings) to be set in the new file.

MERGE concatenates two files (e.g., feelings are combined with traits).

Hardware, language requirements. All the above programs are written in CALL/360-OS FORTRAN, a nonsubset dialect of FORTRAN IV, and currently run in an IBM 370/158. Versions of QUERY, itself, have been written for DEC PDP-11 FORTRAN IV, as well.

Availability. Listings of the CALL/360-OS and DEC PDP-11 versions of QUERY, as well as CALL/360-OS support programs, are available from the authors by sending a stamped selfaddressed envelope to them at The Department of Psychology, Livingston College, Rutgers University, New Brunswick, New Jersey 08903.

\section{REFERENCES}

ROSENBERG, S. New approaches to the analysis of personal constructs in person perception. In J. Cole (Ed.), Nebraska Symposium on Motivation. Lincoln: University Nebraska Press, 1976.

SNeath, P. H. A., \& Sokal, P. R. Numerical taxonomy. San Francisco: Freeman, 1973. 